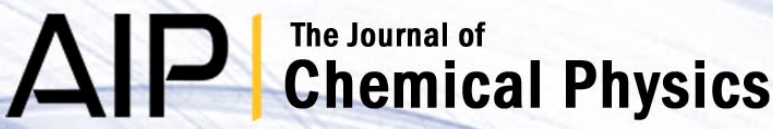

A comparison of quantum and quasiclassical statistical models for reactions of electronically excited atoms with molecular hydrogen

F. J. Aoiz, T. González-Lezana, and V. Sáez Rábanos

Citation: J. Chem. Phys. 129, 094305 (2008); doi: 10.1063/1.2969812

View online: http://dx.doi.org/10.1063/1.2969812

View Table of Contents: http://jcp.aip.org/resource/1/JCPSA6/v129/i9

Published by the AIP Publishing LLC.

Additional information on J. Chem. Phys.

Journal Homepage: http://jcp.aip.org/

Journal Information: http://jcp.aip.org/about/about_the_journal

Top downloads: http://jcp.aip.org/features/most_downloaded

Information for Authors: http://jcp.aip.org/authors

\section{ADVERTISEMENT}

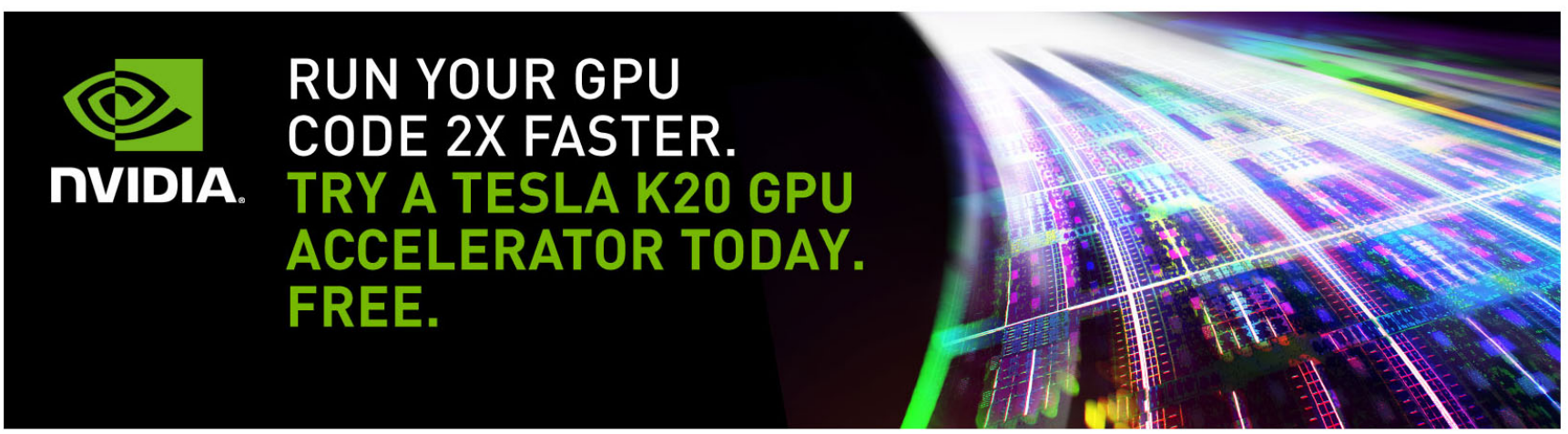




\title{
A comparison of quantum and quasiclassical statistical models for reactions of electronically excited atoms with molecular hydrogen
}

\author{
F. J. Aoiz, ${ }^{1, a)}$ T. González-Lezana, ${ }^{2, b)}$ and V. Sáez Rábanos ${ }^{3, c)}$ \\ ${ }^{1}$ Departamento de Química Física, Facultad de Química, Universidad Complutense, 28040 Madrid, Spain \\ ${ }^{2}$ Instituto de Física Fundamental (CSIC), Serrano 123, 28006 Madrid, Spain \\ ${ }^{3}$ Departamento de Química y Bioquímica. E.T.S. de Ingenieros de Montes, Universidad Politécnica de \\ Madrid, 28040 Madrid, Spain
}

(Received 20 June 2008; accepted 21 July 2008; published online 4 September 2008)

\begin{abstract}
A detailed comparison of statistical models based on the quasiclassical trajectory (SQCT) and quantum mechanical (SQM) methods is presented in this work for the $\mathrm{C}\left({ }^{1} D\right)+\mathrm{H}_{2}, \mathrm{~S}\left({ }^{1} D\right)+\mathrm{H}_{2}$, $\mathrm{O}\left({ }^{1} D\right)+\mathrm{H}_{2}$ and $\mathrm{N}\left({ }^{2} D\right)+\mathrm{H}_{2}$ insertion reactions. Reaction probabilities, integral (ICS) and differential (DCS) cross sections at different levels of product's state resolution are shown and discussed for these reactions. The agreement is in most cases excellent and indicates that the effect of tunneling through the centrifugal barrier is negligible. However, if there exists a dynamical barrier, as in the case of the $\mathrm{N}\left({ }^{2} D\right)+\mathrm{H}_{2}$ reaction, some of the SQM results can be slightly different than those calculated with the SQCT model. The rationale of the observed similarities and discrepancies can be traced back to the specific topologies of the potential energy surfaces for each of the reactions examined. The SQCT model is sensitive enough to show the relatively small inaccuracies resulting from the decoupling inherent to the centrifugal sudden approximation when used in the SQM calculations. In addition, the effect of ignoring the parity conservation is also examined. This effect is in general minor except in particular cases such as the DCS from initial rotational state $j=0$, which requires, in order to reproduce the sharp forward and backward peaks, the explicit conservation of parity. (C) 2008 American Institute of Physics. [DOI: 10.1063/1.2969812]
\end{abstract}

\section{INTRODUCTION}

Over the last few years, the reactions of molecular hydrogen with excited atoms $\mathrm{O}\left({ }^{1} D\right), \mathrm{N}\left({ }^{2} D\right), \mathrm{C}\left({ }^{1} D\right)$, and $\mathrm{S}\left({ }^{1} D\right)$ have attracted a great deal of interest from both the experimental and theoretical points of view. ${ }^{1}$ All these systems feature the presence of a deep well in the ground potential energy surface (PES), associated with the corresponding $\mathrm{AH}_{2}$ stable molecule or radical $\left(\mathrm{H}_{2} \mathrm{O}, \mathrm{NH}_{2}, \mathrm{CH}_{2}\right.$, and $\left.\mathrm{H}_{2} \mathrm{~S}\right)$, and a small or null barrier for insertion. They give rise to strongly bound intermediate complexes which can be deemed as a superexcited molecule or radical.

Accurate, fully converged quantum mechanical (QM) calculations have become available recently for this series of reactions, ${ }^{2-10}$ and have allowed to test the PES for these systems by comparison with highly resolved scattering experiments. ${ }^{4,9,11-15}$ However, exact (meaning accurate, fully converged) QM (EQM) calculations are very demanding computationally and, depending on the particular reaction, are limited to selected isotopic variants in a limited range of small collision energies. For these and other insertion reactions that proceed via formation of intermediate complexes, the various statistical models provide an attractive alternative. Indeed comparisons with EQM calculations and experimental measurements have proved that statistical models constitute a precise global picture of the dynamics of insertion reactions such as those studied in the present work. ${ }^{16}$

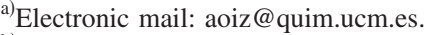

${ }^{\mathrm{b})}$ Electronic mail: tglezana@imaff.cfmac.csic.es.

${ }^{c)}$ Electronic mail: v.saez@upm.es.
}

Statistical approximations applied to the study of chemical reactions were developed in the sixties in the context of phase space theory (PST) by Light and co-workers, ${ }^{17-20}$ and, subsequently, Miller developed a unifying summary of previous works and established some of the key ingredients of modern statistical approaches. ${ }^{21}$ Although since then there has been numerous applications of statistical models, it was not until recently that Manolopoulos and co-workers ${ }^{22,23}$ developed a statistical quantum mechanical (SQM) model to tackle the study of triatomic insertion reactions, which as a novelty included the conservation of triatomic parity. This model has been applied to a variety of reactions whose dynamics is dominated by complex-forming mechanisms. The comparison with EQM calculations has shown that this approximation leads to results in a fairly good agreement with those obtained with rigorous EQM treatments and has proved to be very useful to simulate experimental data with a high level of accuracy. ${ }^{14,22-25}$

In two previous articles, ${ }^{26,27}$ we have presented a statistical quasiclassical trajectory (SQCT) model aimed to overcome some of the shortcomings of the conventional QCT approach. Indeed, such a method complies with the zero point energy in the products, the microscopic reversibility, and explicitly includes parity conservation. The model constitutes a QCT version of the SQM method by Manolopoulos and coworkers with the main difference that in this present approach, instead of wave functions, trajectories are propagated. As a test, the SQCT was applied to obtain reaction probabilities as a function of the total angular momentum $J$, integral cross sections (ICS), and differential cross sections 
(DCS) for the $\mathrm{H}^{+}+\mathrm{H}_{2}$ and $\mathrm{H}^{+}+\mathrm{D}_{2}$ exchange reactions at several collision energies. It was found that the newly developed SQCT approach reproduces the SQM results with an almost perfect agreement even at the state-to-state level of resolution. ${ }^{27}$ Moreover, the observed discrepancies between the results of the two approaches at this level of resolution when the centrifugal sudden approximation (coupled states) is used in the SQM model (SQM-CS) disappear when this approximation is removed and close-coupling SQM calculations (SQM-CC) are performed, thus demonstrating the accuracy of the SQCT method.

As an interesting aspect, the SQCT DCSs for the $\mathrm{H}_{3}^{+}$and $\mathrm{HD}_{2}^{+}$systems exhibit pronounced peaks in the extreme forward and backward scattering directions as sharp as in the EQM (and SQM) calculations and in contrast with those found in conventional QCT calculations. ${ }^{28,29}$ Until recently, the prevailing interpretation attributed this discrepancy between EQM and QCT results to the role of tunneling through the combined long-range centrifugal and dynamical barriers. ${ }^{4,23}$ However, the fact that the SQCT treatment, which is equivalent in all respects to its QM counterpart except for the possible existence of tunneling effects, is capable of reproducing these sharp peaks in the extreme forward and backward directions rules out this hypothesis almost completely, at least for reactions without dynamical barriers. This corroborates the previous work by Larrégaray et $a .^{30}$ wherein it was shown that the attempts of including tunneling in a semiclassical version of the PST failed to account for the existence of such strong polarization in the DCS. Subsequently, Bonnet et al. ${ }^{31,32}$ argued that the reason for the apparent inability of conventional classical treatments to reproduce these sharp peaks near $0^{\circ}$ and $180^{\circ}$ has to be related to the fact that the triatomic parity is ignored in the QCT method. Conservation of triatomic parity in QM implies that $(-1)^{j+l}$ must be the same as $(-1)^{j^{\prime}+l^{\prime}}$, where $l\left(l^{\prime}\right)$ and $j\left(j^{\prime}\right)$ are the orbital and rotational angular momenta of the reagents (products), respectively. Equivalently, in the helicity representations, conservation of parity implies that the zero projection of the absolute helicity quantum number of reagents, $k$, or products, $k^{\prime}-$ i.e., the quantum number of the projection of the total angular momentum $J$ onto the respective atom-diatom internuclear axis-only contributes to the $I=(-1)^{J}$ parity. Although initial $J, l$, and $j$ values (or alternatively $J, j$, and $k$ ) can be quantized in QCT calculations by sampling only discrete integers values, ${ }^{33-35}$ this quantization disappears in the course of the trajectory and the resulting distribution in the products becomes continuous (real values of $l^{\prime}, j^{\prime}$, and $k^{\prime}$ ) and there is not a simple way to enforce the conservation of parity. As mentioned above, conservation of triatomic parity is explicitly included in the SQCT method in the same way as it is in the SQM model. However, a simple modification of the formulation allows to artificially disregard this effect (SQCT-NP), as it has been shown in detail in previous works, ${ }^{26,27}$ and its importance can be addressed rigorously. It was found that the effects of ignoring the triatomic parity are in general minor with two significant exceptions: (i) when the initial diatom rotational state is $j=0$ and/or (ii) near $0^{\circ}$ and $180^{\circ}$ scattering angles. Specifically, it was demonstrated that forward and backward peaks are greatly diminished in the SQCT-NP treatment most especially when $j=0$, and that in order to reproduce the sharpness of these peaks in the DCSs parity conservation has to be included. Thus previous predictions by Bonnet et al. ${ }^{31,32}$ seemed to be fully corroborated in the case of the $\mathrm{H}^{+}$ $+\mathrm{H}_{2} / \mathrm{D}_{2}$ reactions.

In addition to the above discussed failure to describe the sharpness of the forward and backward peaks observed in the DCS of some complex-forming atom-diatom reactions, usual QCT approaches have been found to underestimate the exact total reaction probabilities for large values of $J$ at specific values of the energy for the $\mathrm{H}^{+}+\mathrm{H}_{2}$ (Ref. 28) and $\mathrm{H}^{+}+\mathrm{D}_{2}$ (Ref. 29) reactions. The SQCT approach, on the contrary, is fully consistent with the QM version of the statistical method, ${ }^{26,27}$ and reproduces quite satisfactorily EQM opacity functions for these reactions. ${ }^{27}$ On a recent investigation, ${ }^{36}$ these deficiencies of conventional Gaussian weighted trajectory methods have been attributed to the participation of vibrationally adiabatic nonreactive trajectories in the calculation of the total reaction probabilities. The proposed adiabatic correction leads to QCT probabilities in a noticeably better agreement with the exact results for the $\mathrm{H}^{+}+\mathrm{H}_{2}$ and $\mathrm{H}^{+}+\mathrm{D}_{2}$ reactions.

Comparison between SQCT and SQM has been limited to the barrierless $\mathrm{H}^{+}+\mathrm{H}_{2} / \mathrm{D}_{2}$ reactions. It remains to be seen if a similar degree of agreement is found in the prototypical series of reactions involving excited state atoms and $\mathrm{H}_{2}$. Hence, in this paper, we extend our previous studies and a similar investigation is carried out on the $\mathrm{C}\left({ }^{1} D\right)+\mathrm{H}_{2}$, $\mathrm{S}\left({ }^{1} D\right)+\mathrm{H}_{2}, \mathrm{O}\left({ }^{1} D\right)+\mathrm{H}_{2}$ and $\mathrm{N}\left({ }^{2} D\right)+\mathrm{H}_{2}$ reactions. All these reactions share the presence of a deep well but their respective PESs differ considerably. As will be seen, in some cases the reactions are essentially barrierless for all possible approaches but in others there are even considerable barriers for some ranges of the approaching angle.

The paper is organized as follows: A brief description of the statistical model and details of the calculations is given in Sec. II. The results obtained with the SQCT and SQM models will be presented in Sec. III for each of the four reactions mentioned above, including a comparison with the available EQM results. In Sec. IV the results, and especially the discrepancies between SQCT and SQM results, will be explained in terms of the topologies of each respective PES. We end in Sec. V with a brief summary of the conclusions of this work.

\section{METHOD AND DETAILS OF THE CALCULATIONS}

The formulation of the SQM and SQCT model has been amply described in previous articles. ${ }^{16,23,26,27}$ Only the most relevant aspects will be briefly outlined here.

The statistical model assumes that all reactive collisions proceed via formation of a collision complex whose lifetime is long enough as to treat its formation and decay as independent events. Under these assumptions, the reaction probability at a given total energy $E$, total angular momentum $J$, and a triatomic parity $I( \pm 1)$ from a given reagent state characterized by $v, j$, and $k$, vibrational, rotational, and helicity (the projection of both $\mathbf{J}$ and $\mathbf{j}$ onto the initial relative veloc- 
ity) quantum numbers, respectively, to a final state of the products given by $v^{\prime}, j^{\prime}$, and $k^{\prime}$ can be written as

$$
P_{v^{\prime} j^{\prime} k^{\prime} v j k}^{J, I}(E)=p_{v j k}^{J, I, \alpha} \frac{p_{v^{\prime} j^{\prime} k^{\prime}}^{J, I, \alpha^{\prime}}}{D^{J, I}},
$$

where $p_{v j k}^{J, I, \alpha}$ and $p_{v^{\prime} j^{\prime} k^{\prime}}^{J, I, \alpha^{\prime}}$ are respectively the capture probabilities (the probabilities of forming the collision complex) from the reagent arrangement channel $\alpha$ and from the product arrangement $\alpha^{\prime}$. The quotient of Eq. (1) represents the fraction of collision complexes that decay into the product channel $v^{\prime}, j^{\prime}, k^{\prime}$, where $D^{J, I}$ is given by

$$
D^{J, I}=\sum_{\alpha^{\prime \prime}=1}^{3} \sum_{v^{\prime \prime} j^{\prime \prime} k^{\prime \prime}} p_{v^{\prime \prime} j^{\prime \prime} k^{\prime \prime}}^{J, I, \alpha^{\prime \prime}}
$$

that is, the sum of capture probabilities over the three arrangements and all possible states accessible at a total energy E.

In the SQM version, the capture probability for an arrangement $\alpha$ is given by

$$
p_{v j k}^{J, I, \alpha}(E)=1-\sum_{v^{\prime} j^{\prime} k^{\prime}}\left|S_{v^{\prime} j^{\prime} k^{\prime} v j k}^{J, I, \alpha}(E)\right|^{2},
$$

where the scattering matrix $S$ is calculated separately in each arrangement channel with the standard inelastic scattering form. $^{22}$

The SQCT model uses the $J, j, k$ quantization scheme ${ }^{35}$ based on the discrete sampling of $J, v, j$, and $k$. For each trajectory, once $J$ and $j$ have been selected, the values of $k$ are sampled uniformly and discretely from integers in the interval $[-\min (J, j), \min (J, j)]$. This quantization of $k$ allows the selection of the triatomic parity in a very simple manner.

Trajectories from both reactant's and product's arrangement channels are not integrated until they reach the asymptotic region (with the assignment of a reactive or nonreactive outcome), but only until they have been captured by the potential energy well. A possible choice of the criterion of capture is to characterize complex formation by a given (negative) value of the potential energy $V_{\text {cap }}$, which in QCT calculations is a local property, rather than by defining a capture radius $\left(R_{\text {cap }}\right)$ as in the SQM calculations. Those trajectories experiencing that value have surmounted the centrifugal barrier and are assumed to get trapped in the potential well.

With the above mentioned quantization scheme, the SQCT expression of the capture probability for each channel is given by

$$
p_{v j k}^{J, \alpha}=\frac{N_{c}^{\alpha}(J, v, j, k)}{N^{\alpha}(J, v, j, k)} .
$$

Here, $N^{\alpha}(J, v, j, k)$ and $N_{c}^{\alpha}(J, v, j, k)$ are, respectively, the total number of trajectories and the number of these captured for given values of $J, v, j, k$ in the arrangement channel $\alpha$. The values of $k$ are restricted to integers in the interval $k \in\left[0, k_{\max }\right]$, where $k_{\max }=\min (J, j)$; that is, only the absolute value of $k$ is considered.
The triatomic parity is introduced as in the QM treatment. Of the two possible triatomic parities, $I=(-1)^{J}$ and $I$ $=(-1)^{J+1}, k=0$ only contributes to the former, whereas the $k>0$ values appear in both parities, i.e.,

$$
\begin{aligned}
& p_{v j k}^{J, I, \alpha}=p_{v j k}^{J, \alpha} \quad \text { for } I=(-1)^{J},(-1)^{J+1}, \quad \text { when } k \neq 0 \\
& p_{v j k}^{J, I, \alpha}=p_{v j k}^{J, \alpha} \delta_{I,(-1)^{J}}, \quad \text { when } k=0 .
\end{aligned}
$$

Once the capture probabilities have been calculated using either the QM or the QCT approach, Eq. (1) renders the fully state-to-state parity resolved reaction probability. The various magnitudes, reaction probabilities, integral cross sections, and differential cross section can be readily calculated using the equations of Refs. 23, 26, and 27 and are identical in the QM and QCT cases.

In previous articles, the effect of the parity was thoroughly examined. The expressions when the triatomic parity is disregarded were shown in Refs. 26 and 27. In this modification, that will be denoted by SQCT-NP, the capture probability is given by Eq. (4) with $k=-\min (J, j), \ldots, \min (J, j)$ without exclusion of negative values. The corresponding equations for the various magnitudes can be found in Ref. 27 and they are entirely similar to those when the parity is considered by replacing the summation over $I$ by the summation over negative and positive (including zero) values of the helicity quantum number.

\section{A. Calculation details}

As mentioned above, the reactions studied in this work have in common the fact that their ground PESs are dominated by the existence of deep wells and small or null barriers for perpendicular insertion.

SQM and SQCT calculations have been performed on the same ground adiabatic Born-Oppenheimer PES for each of the reaction here examined. For the $\mathrm{C}\left({ }^{1} D\right)+\mathrm{H}_{2}$ reaction, the $1^{1} A^{\prime}$, the PES by Ho et al. ${ }^{37}$ was used. This PES is a fit using the reproducing kernel Hilbert space (RKHS) interpolation method to the $a b$ initio points calculated by BusseryHonvault et al. ${ }^{5}$ The calculations for the $\mathrm{S}\left({ }^{1} D\right)+\mathrm{H}_{2}$ reaction have been carried out on the $1^{1} A^{\prime}$ RKHS PES. ${ }^{38}$ The widely used $1^{1} A^{\prime}$ DK PES by Dobbyn and Knowles ${ }^{39,40}$ was employed for the calculations of the $\mathrm{O}\left({ }^{1} D\right)+\mathrm{H}_{2}$ system. Finally, the calculations for the exothermic $\mathrm{N}\left({ }^{2} D\right)+\mathrm{H}_{2}$ reaction were performed on the $1^{2} A^{\prime \prime}$ PES by Ho et al. ${ }^{41}$ This is an improved version of the PES by Pederson et al. ${ }^{42}$ which was used in previous calculations using the SQM model. ${ }^{22,23}$

Calculations with both statistical approaches, SQCT and $\mathrm{SQM}$, have been carried out for $\mathrm{C}\left({ }^{1} D\right)+\mathrm{H}_{2}$ at $E_{c}=80$ and $166 \mathrm{meV}$ for the initial diatom states $v=0, j=0,1$; for the $\mathrm{S}\left({ }^{1} D\right)+\mathrm{H}_{2}$ reaction, at a total energy of $0.368 \mathrm{eV}$ for $v=0$ and $j=0$ and $0.382 \mathrm{eV}$ for $v=0$ and $j=1$ corresponding in both cases to a collision energy of $97 \mathrm{meV}$; for $\mathrm{O}\left({ }^{1} D\right)+\mathrm{H}_{2}$ at $E_{c}=56 \mathrm{meV}$ for the $\mathrm{O}_{2}(v=0, j=0)$ initial state. Finally, for $\mathrm{N}\left({ }^{2} D\right)+\mathrm{H}_{2}$ the calculations were performed at a total energy of $0.435 \mathrm{eV}$, which corresponds to $E_{c}=165 \mathrm{meV}$ for $v=0 j$ $=0$ and $E_{c}=150 \mathrm{meV}$ for $v=0, j=1$. 
TABLE I. Parameters of the SQCT and SQM calculations for the $\mathrm{A}+\mathrm{H}_{2}$ and their reverse $\mathrm{H}+\mathrm{AH}$ arrangements. The second and third columns are the values for the capture potential $V_{\text {cap }}$ considered in the SQCT calculation and the average capture radius $R_{\text {cap }}$ with its standard deviation (within parenthesis) associated to the value of $V_{\text {cap. }}$. The fourth column is the number of trajectories. From the fifth to the last column, the values of the capture radius, the maximum value of the radial coordinate, $R_{\max }$ and the largest diatom rotational state $j_{\max }$ employed in the SQM calculation. Distances are given in $\AA$ and energies in $\mathrm{eV}$.

\begin{tabular}{|c|c|c|c|c|c|c|}
\hline & \multicolumn{3}{|c|}{ SQCT } & \multicolumn{3}{|c|}{ SQM } \\
\hline & $V_{\text {cap }}$ & $R_{\text {cap }}$ & \# traject. & $R_{\text {cap }}$ & $R_{\max }$ & $j_{\max }$ \\
\hline $\mathrm{C}+\mathrm{H}_{2}$ & -0.8 & $1.42(0.04)$ & $1.5 \times 10^{6}$ & 2.88 & 10.08 & 7 \\
\hline $\mathrm{H}+\mathrm{CH}$ & -1.2 & $1.93(0.11)$ & $2.0 \times 10^{6}$ & 2.12 & 7.42 & 19 \\
\hline $\mathrm{S}+\mathrm{H}_{2}$ & -0.6 & $1.84(0.09)$ & $1.0 \times 10^{6}$ & 2.58 & 8.84 & 10 \\
\hline $\mathrm{H}+\mathrm{SH}$ & -0.8 & $2.26(0.28)$ & $2.0 \times 10^{6}$ & 2.33 & 6.35 & 24 \\
\hline $\mathrm{O}+\mathrm{H}_{2}$ & -0.6 & $1.61(0.06)$ & $3.0 \times 10^{6}$ & 2.76 & 10.17 & 8 \\
\hline $\mathrm{H}+\mathrm{OH}$ & -0.6 & $1.72(0.38)$ & $1.5 \times 10^{6}$ & 1.14 & 7.41 & 34 \\
\hline $\mathrm{N}+\mathrm{H}_{2}$ & -0.6 & $1.54(0.06)$ & $1.0 \times 10^{6}$ & 1.81 & 10.13 & 7 \\
\hline $\mathrm{H}+\mathrm{NH}$ & -0.8 & $1.71(0.19)$ & $3.0 \times 10^{6}$ & 1.59 & 7.42 & 30 \\
\hline
\end{tabular}

Table I collects the values of the capture parameters (SQCT, SQM) and the number of trajectories calculated for each of these reactions.

In all cases, for the SQCT calculations, tests of convergence were performed for these reactions varying $V_{\text {cap }}=$ -0.4 to $-1.8 \mathrm{eV}$ with exactly the same results. Analogously, the convergence of the SQM calculations was checked by modifying the corresponding $R_{\text {cap }}$ values. With the only exception of the $\mathrm{H}+\mathrm{OH}$ and $\mathrm{H}+\mathrm{NH}$ arrangements, the $R_{\text {cap }}$ radius in the SQM calculation turns out to be larger than the effective value considered in the SQCT approach; however, considering the insensitivity of the SQCT to the specific value of $V_{\text {cap }}$ in a relatively broad range of negative potentials, the two sets of $R_{\text {cap }}$ are fully compatible.

Except when stated on the contrary, all the SQM calculations have been performed using the full close coupling treatment (SQM-CC). In one case results are also shown using the centrifugal sudden (coupled states) approximation (SQM-CS) to prove the limitations of this method for state resolved magnitudes.

\section{RESULTS}

In this section the results obtained using the QCT and QM versions of the statistical model will be presented for a series of insertion reactions. Basically, three types of reactions have been considered: reactions with a potential barrier at certain angles other than right angles, $\mathrm{C}\left({ }^{1} D\right)+\mathrm{H}_{2}$ and $\mathrm{S}\left({ }^{1} D\right)+\mathrm{H}_{2}$; a reaction with essentially no potential barrier for any possible approaching angle, $\mathrm{O}\left({ }^{1} D\right)+\mathrm{H}_{2}$, and, a predominantly insertion reaction but still with a small barrier for perpendicular attack, $\mathrm{N}\left({ }^{2} D\right)+\mathrm{H}_{2}$, which grows for collinear attack. As will be seen, the $\mathrm{S}\left({ }^{1} D\right)+\mathrm{H}_{2}$ reaction exhibits a late collinear barrier with some interesting features in the entrance channels which will be examined in detail in Sec. IV.

The main goal is to compare the SQM and SQCT reaction probabilities, integral and differential cross sections at different levels of resolution. In addition, the effect of the considering or neglecting the triatomic parity conservation will also be examined in some cases.

\section{A. $C\left({ }^{1} D\right)+\mathrm{H}_{2}$ reaction}

This reaction constitutes a prototype of insertion reaction involving only neutrals. It has a deep well of $4.33 \mathrm{eV}$ with respect to the $\mathrm{C}\left({ }^{1} D\right)+\mathrm{H}_{2}$ dissociation channel and is slightly exothermic by $\Delta H_{0}^{0}=-0.263 \mathrm{eV}(-0.168 \mathrm{eV}$ excluding the zero point energy). It has a steep barrier for collinear approach $\left(0.54 \mathrm{eV}\right.$, located at $\left.R_{\mathrm{H}_{2}}=0.98 \AA, R_{\mathrm{CH}}=1.3 \AA\right)$, while is barrierless for $C_{2 v}$ insertion.

Numerous QM and QCT calculations have clearly proved that this reaction takes place via formation of long lived complexes which give rise to very narrow and dense resonances in the QM reaction probability as a function of $E,{ }^{5,6}$ and to very broad distributions of collision times in QCT calculations with tails extending up to $5 \mathrm{ps}^{1}{ }^{1} \mathrm{QM}$ and QCT DCSs are typically backward-forward symmetric, but the QM results lead to very sharp peaks at $0^{\circ}$ and $180^{\circ}$ for initial $j=0$ (and to a less extent for $j=1$ ) which cannot be reproduced by QCT calculations. As expected, this reaction is ideally suited for statistical treatments, and most of its dynamics is very well reproduced by the SQM model. ${ }^{13,16,23,43}$ In what follows a comparison between SQCT and SQM results will be shown for this reaction.

In the present work SQCT and SQM calculations have been made at four total energies corresponding to initial $v$ $=0, j=0,1$ states and $E_{c}=80$ and $166 \mathrm{meV}^{13}$ The corresponding ICS into $v^{\prime}=0, j^{\prime}$ are shown in Fig. 1 at these collision energies and initial states. As can be seen, the agreement between the SQM-CC and SQCT calculations is very good for the collision energies and initial states here considered. The discrepancies are, however, apparent with the data obtained using the CS approximation. Near the maxima of the rotational distributions, the $v^{\prime}=0, j^{\prime}$ ICSs are systematically larger than those obtained with the SQCT and SQM-CC treatments, while for higher $j^{\prime}$ states the ICS are systematically smaller.

Figure 2 depicts the state-to-state reaction probability as a function of $J, P_{0 j^{\prime}, 00}^{J}$, for $v^{\prime}=0$ and $j^{\prime}=0,10$, and 13 for which the SQM-CS ICSs are the same, larger and smaller, respectively, than those obtained with the SQCT and SQM-CC treatments. For $j^{\prime}=0$ the three sets of results are 

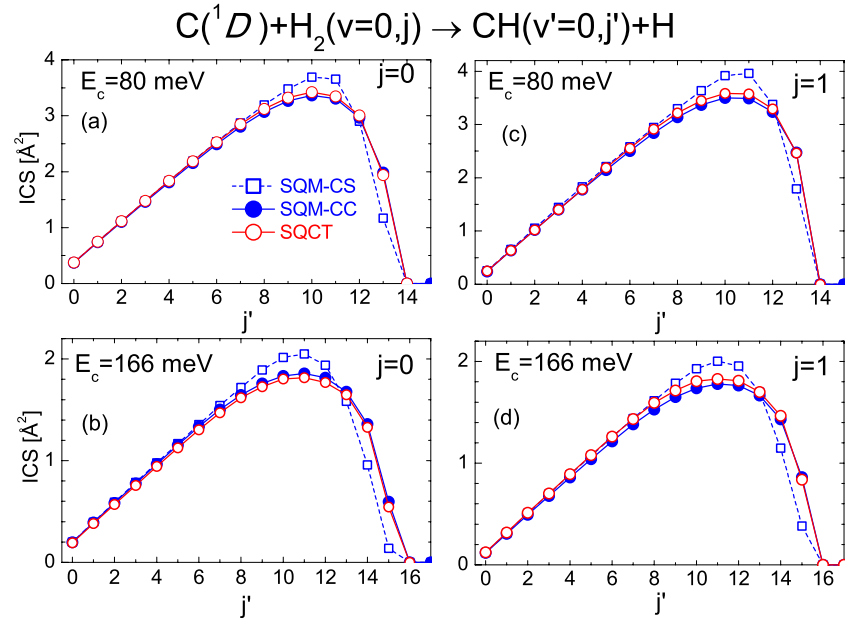

FIG. 1. (Color online) Rotationally resolved integral cross sections $\left(\AA^{2}\right)$ for the $\mathrm{C}\left({ }^{1} D\right)+\mathrm{H}_{2}(v=0, j) \rightarrow \mathrm{CH}\left(v^{\prime}=0, j^{\prime}\right)+\mathrm{H}$ reaction at the indicated collision energies and initial $j$. Open circles: SQCT results. Solid circles: SQMCC. Open squares and dashed line: SQM-CS.

practically coincident. However, the discrepancies are conspicuous for $j^{\prime}=10$ and especially for $j^{\prime}=13$. For $j^{\prime}=10$, which corresponds to the maximum of the rotational distribution [Fig. 1(a)], the SQM-CS and SQCT probabilities agree well up to $J=20$, but for higher $J$ values the SQM-CS $P^{J}$ has a peak and then decreases rapidly to zero, while that using SQCT is smoother and extends to larger values of $J$. For $j^{\prime}=13$, the SQM reaction probability dies out much sooner than the SQCT one and only at the first $J$ values they

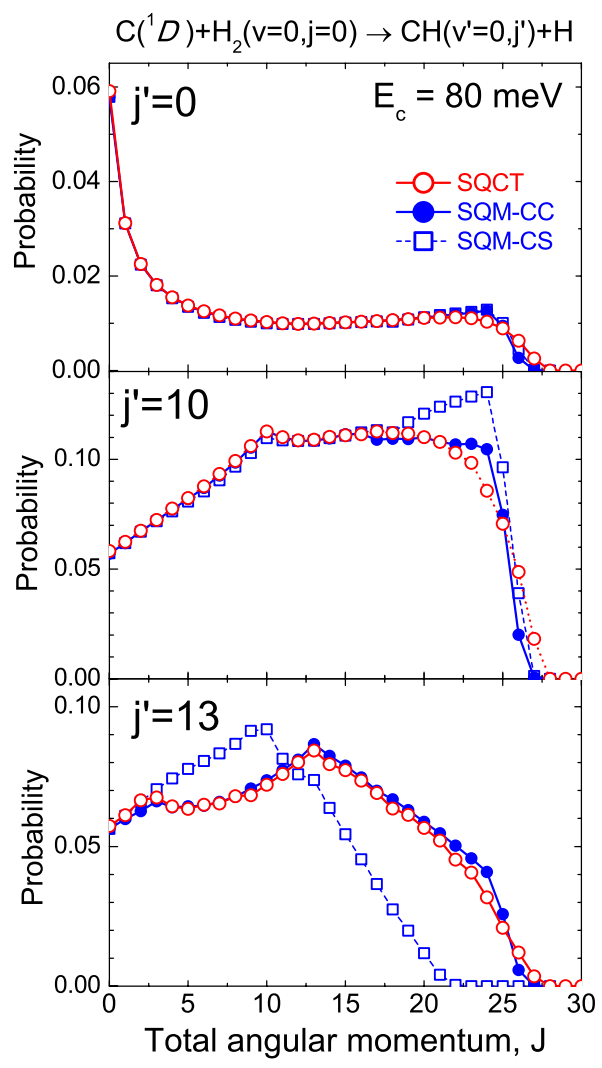

FIG. 2. (Color online) State-to-state reaction probability for the $C\left({ }^{1} D\right)$ $+\mathrm{H}_{2}(v=0, j=0) \rightarrow \mathrm{CH}\left(v^{\prime}=0, j^{\prime}\right)+\mathrm{H}$ reaction at $E_{c}=80 \mathrm{meV}$ for the indicated $j^{\prime}$ states. Symbols as in Fig. 1.

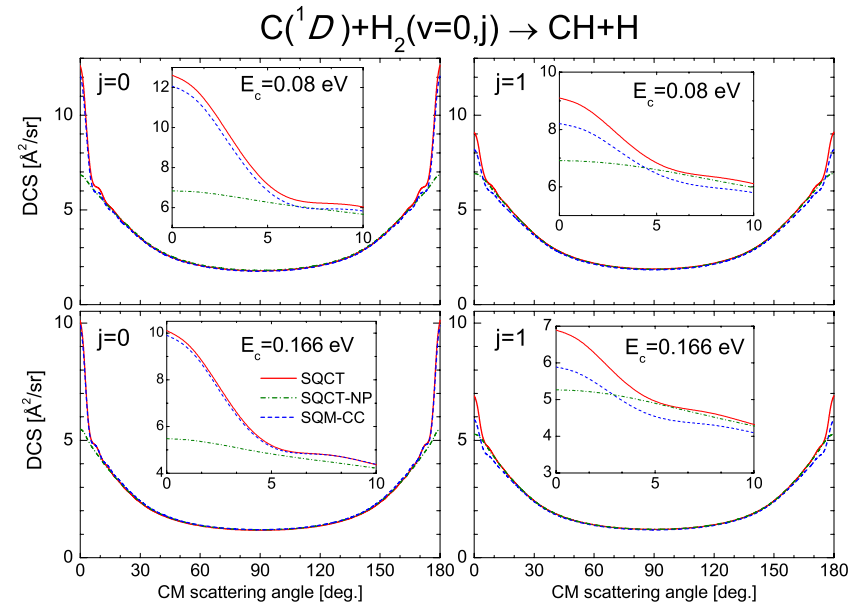

FIG. 3. (Color online) Initial-state-selected differential cross sections $\left(\AA^{2} \mathrm{sr}^{-1}\right)$ summed over all final states for the $\mathrm{C}\left({ }^{1} D\right)+\mathrm{H}_{2}(v=0, j=0,1)$ reaction at the indicated collision energies. Solid line: SQCT. Dashed line: SQM-CC. Dot-dash line: SQCT-NP.

agree with each other. The analysis of the results indicates that the reason of this discrepancy can be traced back to the SQM-CS capture probabilities for high $j^{\prime}$ and $J$ values in the $\mathrm{H}+\mathrm{CH}$ channel, which are smaller than the SQCT ones.

Similar effects were also found in the case of the $\mathrm{H}^{+}$ $+\mathrm{H}_{2}$ and $\mathrm{H}^{+}+\mathrm{D}_{2}$ reactions, ${ }^{27}$ and, as in the carbon reaction, when the CS approximation is lifted, the agreement between SQCT and SQM results became almost perfect. The CS approximation ${ }^{44-47}$ has been used in most of the previous applications of the SQM method with the rationale that the accuracy of the CS approximation must be guaranteed in the SQM calculations since the Coriolis coupling is expected to be minor in the asymptotic regions in which the wave function is propagated. ${ }^{22-24,43}$ However, despite centrifugal decoupling approximations may render accurate results for low $J$ and $j^{\prime}$ values, differences are expected to become progressively more significant as $J$ and $j^{\prime}$ increases, since the centrifugal barrier is overestimated. This was clearly shown in the case of inelastic scattering. ${ }^{46}$ The present results, as well as those obtained for the $\mathrm{H}_{3}^{+}$and $\mathrm{HD}_{2}^{+}$reactive systems, ${ }^{27}$ corroborate the limitations of the CS approximation, at least when final state resolution is achieved. In the remaining of this work, the comparison between SQCT and SQM will be restricted to calculations using the accurate $\mathrm{CC}$ method.

The comparison of SQCT and SQM total DCS for the same initial states and $E_{c}$ is portrayed in Fig. 3. Both sets of data are practically coincident in the whole range of scattering angles, although the SQCT calculations predict a slightly larger value of the DCS near $0^{\circ}$ and $180^{\circ}$, especially for $j=1$. These results agree well with the EQM calculations that were performed in a previous fit of PES based on the same set of $a b$ initio points. It is noticeable that the height of the forward and backward peaks are reproduced by the SQCT results in contrast to what is found in conventional QCT calculations. ${ }^{6}$ Actually, since the expressions of the DCS are identical in both SQM and SQCT models, the good agreement is due to the accordance between $\mathrm{QM}$ and QCT capture probabilities. In their PST calculation on the $\mathrm{C}\left({ }^{1} D\right)+\mathrm{H}_{2}$ reaction, Bonnet et al. ${ }^{31}$ showed that neglect of the parity con- 
servation in PST leads to DCS with forward and backward peaks similar to those found in classical PST. In fact, the SQCT-NP calculations, also shown in Fig. 3, confirm that when triatomic parity is disregarded the scattering at extreme forward and backward angles is considerably smaller than when it is taken into account. This effect was amply described and justified in detail in our previous work on the also barrierless reaction of $\mathrm{H}^{+}+\mathrm{H}_{2} / \mathrm{D}_{2}$. As was demonstrated there, this effect diminishes rapidly as initial $j$ increases, as is also shown in the right panels, corresponding to $j=1$.

\section{B. $S\left({ }^{1} D\right)+\mathrm{H}_{2}$ reaction}

The $\mathrm{S}\left({ }^{1} D\right)+\mathrm{H}_{2}$ reaction is slightly more exoergic $\Delta H_{0}^{0}$ $=-0.286 \mathrm{eV}\left(\Delta D_{e}=0.181 \mathrm{eV}\right)$ than the $\mathrm{C}\left({ }^{1} D\right)+\mathrm{H}_{2}$ reaction. For the collinear approach, $C_{\infty_{v}}$, the calculated PES for this reaction $^{38}$ also exhibits a late barrier whose height is $\approx 0.355 \mathrm{eV}\left(R_{\mathrm{H}_{2}}=0.98 \AA, R_{\mathrm{SH}}=1.52 \AA\right)$. For a range of $\gamma$ angles around the perpendicular attack the PES is barrierless. The specific shape of the PES when the $\mathrm{S}$ excited atom approaches the $\mathrm{H}_{2}$ molecule will be discussed in Sec. IV.

Previous QM and QCT calculations ${ }^{7,8}$ have shown that the reaction takes place via the formation of long-lived complexes with a broad distribution of collision times which extend up to 4-5 ps (Refs. 1 and 8) somewhat larger than those obtained for the $\mathrm{C}\left({ }^{1} D\right)+\mathrm{H}_{2}$ reaction. This is also manifested in a dense resonance structure that appears in the EQM calculations. Hence, this reaction is also expected to be an excellent candidate for a statistical treatment. In fact, previous calculations of total reaction probabilities and DCS using the time independent ${ }^{22}$ and the wave packet ${ }^{48}$ versions of the SQM-CS model were found to be in good agreement with EQM carried out on the same PES. ${ }^{7}$ It remains to be seen if the SQCT can give an accurate description of this reaction similar to that obtained with the SQM model.

The state resolved integral cross sections for the first two rotational states of the $\mathrm{H}_{2}$ molecule are shown in Fig. 4 at $97 \mathrm{meV}$ collision energy, which corresponds to the lowest one of the experiments of Lee and Liu ${ }^{15}$ The rotational distributions calculated with the SQCT and SQM-CC models are also compared with the exact time independent QM results on the same PES for the two vibrational states of the $\mathrm{SH}$ product accessible at this energy. As can be seen, the two statistical treatments constitute a good approximation to the EQM data, although some differences are appreciable. For $v^{\prime}=0$, the cross sections are somewhat overestimated by the SQM and SQCT approaches, while for $v^{\prime}=1$, the opposite takes place. The oscillations found in the EQM data, especially for $j=0$, are absent in the calculations using either of the statistical models.

In general terms, the agreement between SQM-CC and SQCT results is very good with discrepancies in the values of the state-to-state ICS no larger than a few percent. In particular, for $v^{\prime}=1$ the differences are negligible. For $v^{\prime}$ $=0$, there are slight discrepancies especially for the rotational states near the maxima of the respective distributions. While the SQCT cross sections are somewhat underestimated in the case of initial $j=0$, the opposite takes place when $j=1$. Correspondingly, the $v^{\prime}=0$ cross section summed over final ro-
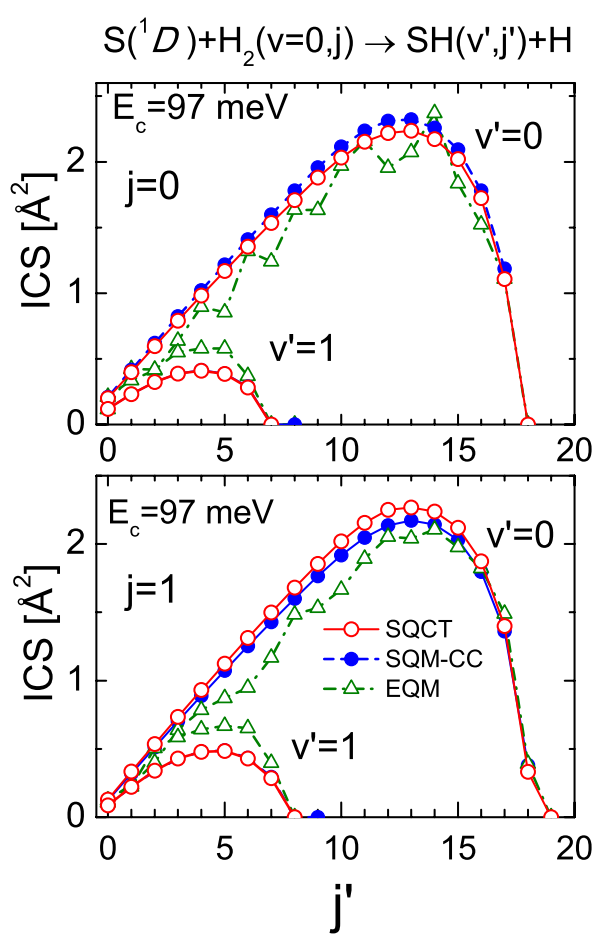

FIG. 4. (Color online) Rotationally resolved ICS $\left(\AA^{2}\right)$ for the $\mathrm{S}\left({ }^{1} D\right)$ $+\mathrm{H}_{2}(v=0, j) \rightarrow \mathrm{SH}\left(v^{\prime}=0,1, j^{\prime}\right)+\mathrm{H}$ reaction at $97 \mathrm{meV}$ collision energy and initial $j=0,1$. Open circles (red): SQCT results. Solid circles (blue): SQMCC. Open triangles (dark green): EQM calculations from Ref. 8. The SQM results for $v^{\prime}=1$ are indistinguishable to those from SQCT calculations.

tational states are slightly larger for $j=0$ in the case of the SQM-CC calculations, while for $j=1$ are smaller.

In order to trace back the discrepancies found in the $v^{\prime}$ $=0$ SQCT and SQM-CC rotational distributions, it is pertinent to examine the vibrationally state resolved opacity functions which are displayed in Fig. 5 for the two initial rotational states of the $\mathrm{H}_{2}$ molecule. The origin of the differences found in the ICS can be readily assigned to the contribution of a particular set of total angular momenta. Until $J=23$, the results obtained with SQCT and SQM-CC are indistinguishable for both $j=0$ and $j=1$. In particular, the SQCT and SQM $P_{v^{\prime}=1,0 j}^{J}$ are practically identical as the highest contribution from $J$ to $v^{\prime}=1$ is $\approx 24$. For higher values of $J$, the SQCT $v^{\prime}=0$ reaction probability from initial $j=0$ has a dip which is not found in the SQM results. In contrast, for initial $j=1$, the SQM reaction probabilities in the $J=25-28$ are smaller than in the QCT version of the model. This explains why the $j$ $=0$ state resolved ICS are larger in the SQM case and the contrary takes place for $j=1$. The analysis of the results for $j=0$ shows that the difference is due to the capture probabilities, $p_{000}^{J}$, which display a dip not found in the SQM-CC calculations. Apparently, in that particular interval of high $J$, the trajectories experience a hindrance which decrease the corresponding reaction probabilities while in the QM calculations such barrier is more easily overcome. A full discussion of this effect will be postponed to Sec. IV.

The total, summed over final states DCS is shown in Fig. 6. The results from the SQCT and SQM-CC calculations are shown together with the EQM results from Ref. 8. The agreement between the three sets of results is very good with the statistical predictions accounting very well for the EQM 

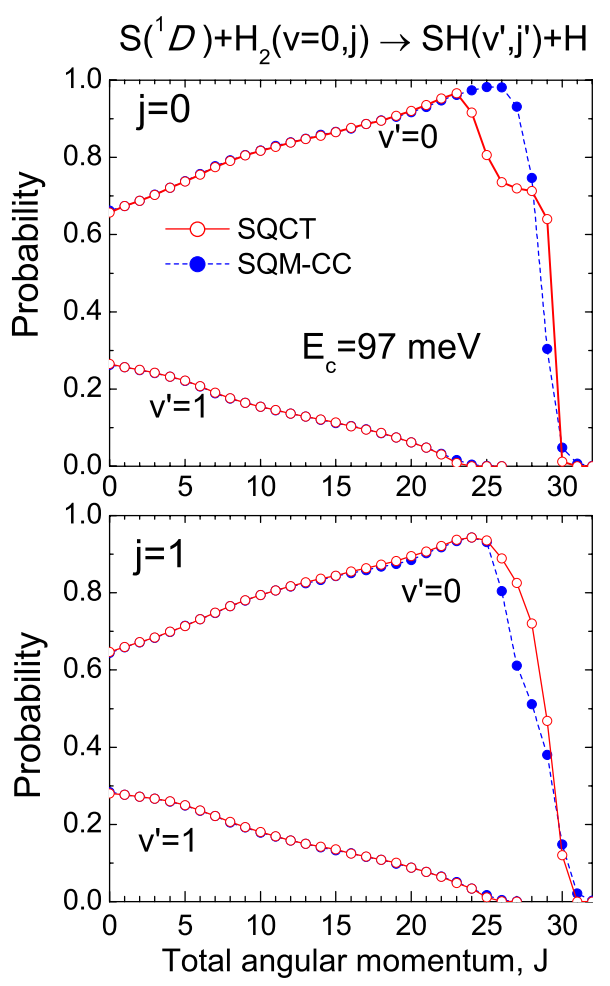

FIG. 5. (Color online) Vibrationally state resolved reaction probability for the $\mathrm{S}\left({ }^{1} D\right)+\mathrm{H}_{2}(v=0, j=0,1) \rightarrow \mathrm{SH}\left(v^{\prime}=0,1\right)+\mathrm{H}$ reaction at $E_{c}=97 \mathrm{meV}$. Open circles (red): SQCT results. Solid circles (blue): SQM-CC.

data. Only for $j=0$, the EQM DCS is slightly asymmetric with a forward peak smaller than the backward one. The SQCT and SQM-CC angular distributions are in a remarkably good accord, and the scattering at $0^{\circ}$ (and thus at $180^{\circ}$ ) is almost identical. A detailed inspection shows that for $j$ $=0$ in the $10^{\circ}-60^{\circ}$ (and thus $120^{\circ}-170^{\circ}$ ) range of scattering angles, the magnitude of SQM DCS is slightly larger than that from the SQCT calculations, while the opposite happens for $j=1$. In any case, at the resolution of the plot, the small effect observed in the state resolved ICS and reaction probabilities is practically washed out when summing over final states. This is not surprising since as it was already shown, ${ }^{8}$ and can be also demonstrated with the present data, all $J$ contribute to scattering over the whole angular range.

\section{C. $\mathrm{O}\left({ }^{1} D\right)+\mathrm{H}_{2}$ reaction}

The $\mathrm{O}\left({ }^{1} D\right)+\mathrm{H}_{2}$ is probably the best studied insertion reaction both from the experimental and theoretical points of view. In contrast to the two previous reactions examined up to this point, it is strongly exothermic $\left(\Delta D_{e}=1.88 \mathrm{eV}\right)$ and at small collision energies $\mathrm{OH}$ vibrational states up to $v^{\prime}=4$ are populated. Although several PESs correlating with the reagents can contribute either adiabatically or nonadiabatically to the reaction, the global reactivity is dominated by the essentially barrierless $1{ }^{1} A^{\prime}$ ground PES. Moreover, the excited $1{ }^{1} A^{\prime \prime}$ and $2{ }^{1} A^{\prime}$ PESs exhibit a minimum collinear barrier of $\approx 0.10 \mathrm{eV}$, and for collision energies below that value their contribution can be neglected without introducing any significant error. ${ }^{12}$

In this work, calculations are restricted to the ground $1{ }^{1} A^{\prime}$ DK PES. ${ }^{39}$ at $E_{\mathrm{c}}=56 \mathrm{meV}$, which correspond to the
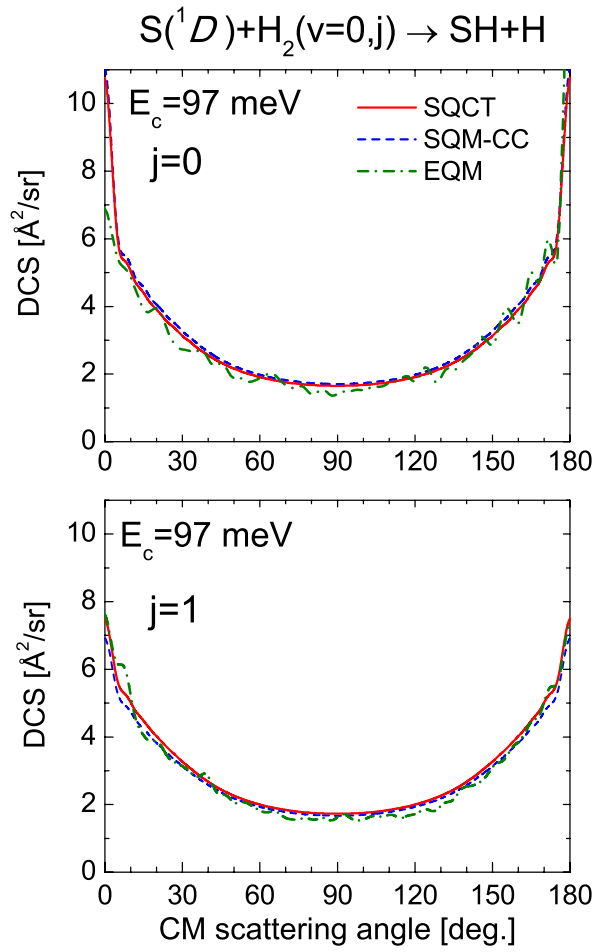

FIG. 6. (Color online) Initial-state-selected DCS $\left(\AA^{2} \mathrm{sr}^{-1}\right)$ summed over all final states for the $\mathrm{S}\left({ }^{1} D\right)+\mathrm{H}_{2}(v=0, j=0,1)$ reaction at $97 \mathrm{meV}$ collision energies. Solid line: SQCT. Dashed line: SQM-CC. Dot-dash line: EQM results from Ref. 8.

collision energy of the experiments of Yang and coworkers ${ }^{12}$ on the ground As mentioned above, this PES is barrierless for most of the skewed configurations. Only for collinear approach there is a small early barrier of $\approx 10 \mathrm{meV}\left(R_{\mathrm{H}_{2}}\right.$ $=0.76 \AA, R_{\mathrm{OH}}=1.7 \AA$ ). In addition, this PES has a very deep well of $7.29 \mathrm{eV}$ for a $C_{2 v}$ geometry measured from the asymptotic reactant's valley.

For this reaction, the collision complex is formed with much energy in excess of its dissociation limit into products, and thus can break apart more easily than in the reactions of $\mathrm{C}\left({ }^{1} D\right)$ and $\mathrm{S}\left({ }^{1} D\right)$ with $\mathrm{H}_{2}$. The distribution of classical collision times spans a relatively short range of times, peaking at $100 \mathrm{fs}$ and with a tail extending no further than $400 \mathrm{fs}{ }^{19}$ This is also confirmed by the relatively structureless EQM reaction probability as a function of energy, indicative of short lived intermediates. In spite of the relatively short lifetime of the collision complexes, the results obtained with the SQM-CS proved to be in a fairly good accordance with the exact results. We will examine several reaction magnitudes comparing the SQCT, SQM, and, when available, the EQM results, all of them using the same PES.

The $v^{\prime}=0,4$ rotational distributions for $v=0, j=0$ obtained with the three methods are represented in Fig. 7. The results obtained at this level of resolution with the SQCT and $\mathrm{SQM}$ are in excellent agreement, similar to that shown for the $\mathrm{C}\left({ }^{1} D\right)+\mathrm{H}_{2}$. This agreement is common to all the $\mathrm{OH}$ vibrational states. There are, however, significant differences with the rotational distributions resulting from the EQM treatment, especially for $v^{\prime}=0$. As $v^{\prime}$ increases the agreement improves. This comparison suggests that the rotational distributions become more statistical with increasing $v^{\prime}$. In 


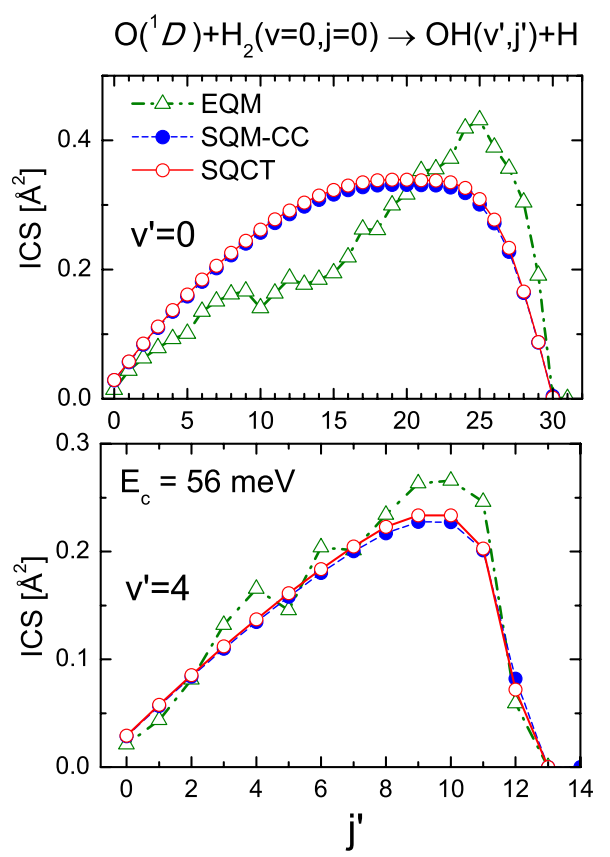

FIG. 7. (Color online) Rotationally resolved ICS $\left(\AA^{2}\right)$ for the $\mathrm{O}\left({ }^{1} D\right)$ $+\mathrm{H}_{2}(v=0, j=0) \rightarrow \mathrm{OH}\left(v^{\prime}=0,4, j^{\prime}\right)+\mathrm{H}$ reaction at $56 \mathrm{meV}$ collision energy. Open (red) circles: SQCT results. Solid (blue) circles: SQM-CC. Open (green) triangles: exact QM calculations from Ref. 3.

fact, previous QCT calculations on this reaction ${ }^{49}$ have shown that the collision times increase with increasing $v^{\prime}$ at $100 \mathrm{meV}$ collision energy. The average lifetime of the complex calculated there for $v^{\prime}=4$ is almost three times larger than for $v^{\prime}=0$.

SQCT and SQM-CC state-to-state reactions probabilities into $v^{\prime}=0, j^{\prime}=0,10$, and 20 are compared in Fig. 8. In all cases, the results are in excellent accordance. Only at the highest $J$ values some small discrepancies are visible. Similar degree of agreement is found for the vibrationally resolved and the total reaction probabilities (not shown). As in the case of the $\mathrm{C}\left({ }^{1} D\right)+\mathrm{H}_{2}$ reaction, the use of the CS approximation yields results at variance with those shown in the figure.

Finally, the total DCSs calculated with the SQCT and SQM-CC methods are shown in Fig. 9. The respective curves are almost identical with tiny differences at sideways scattering angles, where the SQCT DCS is slightly larger then the SQM-CC DCS. For comparison purposes, the EQM DCS is also portrayed in the figure. Although the statistical methods account for the EQM DCS fairly well, scattering in the forward hemisphere is somewhat underestimated while the opposite happens with scattering in backward hemisphere.

\section{D. $\mathrm{N}\left({ }^{2} D\right)+\mathrm{H}_{2}$ reaction}

The ground $1{ }^{2} A$ " PES for this reaction exhibits a very deep well of $5.48 \mathrm{eV}$. In contrast to the previous systems, this PES has a barrier in the entrance channel for all interatomic orientations. Specifically, for a $C_{2 v}$ approach, it has a small barrier of $78 \mathrm{meV}$ which increases towards the linear configuration. For collinear approach, the barrier is located at $R_{\mathrm{H}_{2}} \approx 0.8 \AA$ and $R_{\mathrm{NH}} \approx 1.5 \AA$, with a height of $210 \mathrm{meV}$. As

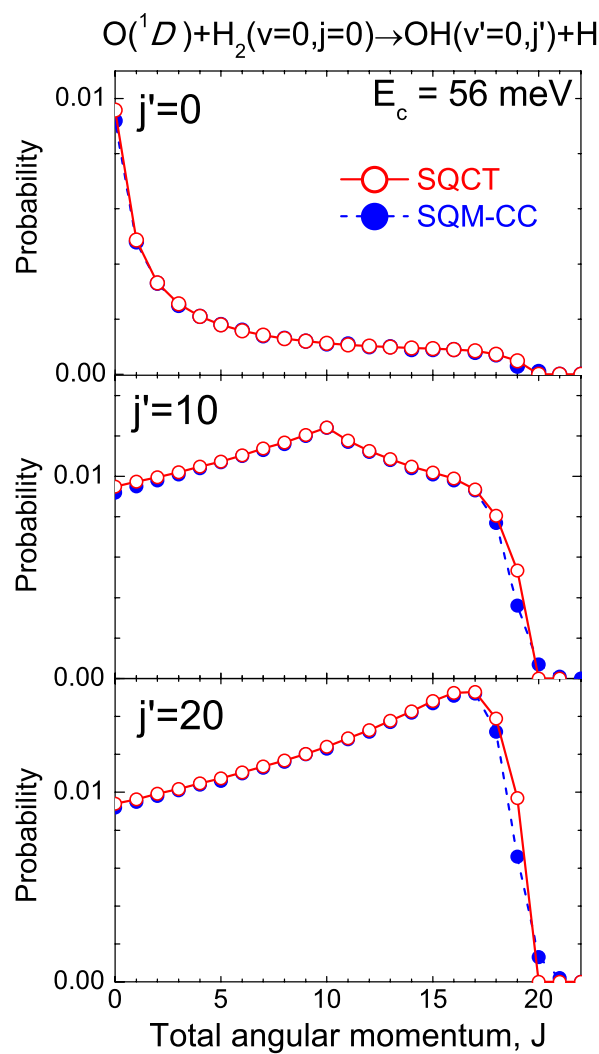

FIG. 8. (Color online) State-to-state reaction probability for the $\mathrm{O}\left({ }^{1} D\right)$ $+\mathrm{H}_{2}(v=0, j=0) \rightarrow \mathrm{OH}\left(v^{\prime}=0, j^{\prime}\right)+\mathrm{H}$ reaction at $E_{c}=56 \mathrm{meV}$ for $j^{\prime}$ $=0,10,20$. Symbols as in Fig. 2 .

in the case of the $\mathrm{O}\left({ }^{1} D\right)+\mathrm{H}_{2}$ reaction, the reaction is highly exothermic $\left(\Delta D_{e}=1.254 \mathrm{eV}\right)$ allowing the population of many rovibrational states in the $\mathrm{NH}$ product channel.

Also in common with the reaction with $\mathrm{O}\left({ }^{1} D\right)$ reaction, the collision complexes are formed with much energy in excess of their dissociation limit in the product channel and have an appreciable shorter average lifetime than those formed in the almost thermoneutral reactions previously examined. The distribution of classical collision times at $E_{\mathrm{c}}$ $=165 \mathrm{meV}$ is quite narrow extending no much further than

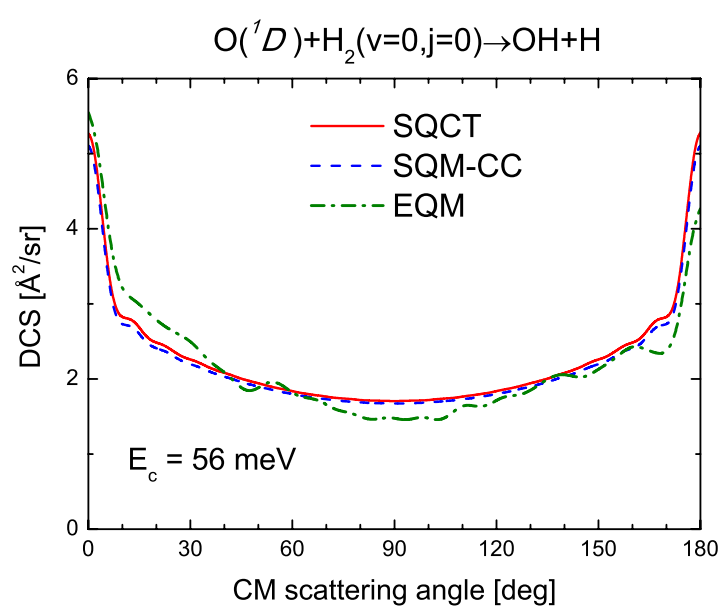

FIG. 9. (Color online) Total (summed over final states) DCS for the $\mathrm{O}\left({ }^{1} D\right)+\mathrm{H}_{2}(v=0, j=0) \rightarrow \mathrm{OH}+\mathrm{H}$ reaction at $E_{c}=56 \mathrm{meV}$. Solid (red) line: SQCT; dashed (blue) line: SQM-CC; dot-dash (green) line: EQM results from Ref. 3. 


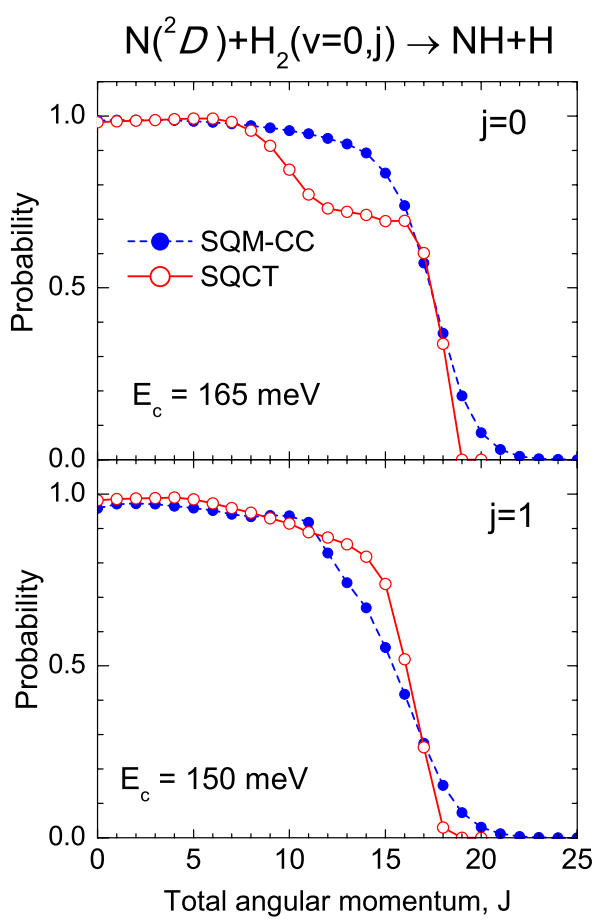

FIG. 10. (Color online) Total (summed over final states) reaction probability for the $\mathrm{N}\left({ }^{2} D\right)+\mathrm{H}_{2}(v=0, j) \rightarrow \mathrm{NH}+\mathrm{H}$ reaction at $E_{c}=165 \mathrm{meV}$ and $j=0$ (top) and $E_{c}=150 \mathrm{meV}$ and $j=1$ (bottom). Open (red) circles and solid line: SQCT. Solid (blue) circles and dashed line: SQM-CC.

$300 \mathrm{fs}$. This is mirrored in the smoother QM reaction probabilities and in less symmetric DCSs. Nevertheless, the main dynamical features are well captured by the SQM model and the reaction probabilities, rotational distributions, and DCS are fairly well accounted for by the SQM model. ${ }^{23,25}$ As in the previous reactions, the QM and SQM DCS display sharp forward and backward peaks which are missed in the QCT calculations.

SQM and SQCT calculations have been carried out at the total energy $E_{\text {tot }}=434 \mathrm{meV}$ which, for the initial $v=0, j$ $=0$ state, corresponds to $E_{\mathrm{c}}=165 \mathrm{meV}$, identical to the mean collision energy of the experiments of Balucani et al. ${ }^{4,25} \mathrm{Cal}-$ culations are also shown at the same total energy for $v=0$, $j=1$, which corresponds $E_{\mathrm{c}}=150 \mathrm{meV}$.

Figure 10 portrays the comparison of the SQM-CC and SQCT total reaction probability at the two collision energies and initial states. At low values of $J$ the reaction probability is close to one, and the two sets of calculations are almost indistinguishable. However, at $10<J<17$ the differences become clearly apparent. For initial $j=0$ the SQCT $P^{J}$ has a pronounce dip which is absent in the SQM results. For initial $j=1$, although the agreement is somewhat better, the SQM reaction probability decreases in this range of $J$ more rapidly than its SQCT counterpart. The situation is remarkably similar to that found in the $\mathrm{S}\left({ }^{1} D\right)+\mathrm{H}_{2}$ reaction, but is still more marked. As in that case, the discrepancies for $j=0$ can be attributed to the differences in the capture probabilities which are smaller in the SQCT calculations in that range of total angular momenta.

In addition, at the highest values of $J$ leading to reaction, the SQM $P^{J}$ displays a tail up to $J=22$, while the SQCT probability becomes abruptly zero at $J=19$. This effect, which is absent in the other reactions examined so far, is due to tunneling through the combined dynamical and centrifugal barriers, as shown in previous EQM. ${ }^{50}$ Although the barrier for insertion is rather small $(\approx 78 \mathrm{meV})$, it is sufficiently effective for collisions at the highest $J$ with a small remnant of radial energy. This barrier, however, can be crossed in the QM case.

These discrepancies are expected to be reflected in the DCS, and this is indeed the case as can be seen in Fig. 11. The agreement between the SQM and SQCT is clearly worse than that found for the other reactive systems. For $j=0$ (top panel), apart from the differences in extreme forward and backward scattering angles, it is noticeable the fact that sideways scattering is underestimated in the SQCT calculations and hence the total reaction cross section is also somewhat smaller.

For comparison purposes, EQM results from Ref. 2 are also represented in this figure. It should be warned that these EQM calculations have been carried out on the original PES by Pederson et al. ${ }^{42}$ which has a slightly higher barrier than that used in the present work. ${ }^{41}$ In spite of this, the differences between EQM DCS on both PESs are relatively small $^{10}$ and constrained to the backward region. In the EQM calculations, the DCS is somewhat asymmetric with a backward peak higher than that found near $0^{\circ}$ and has some oscillations. The lack of perfect symmetry reveals, as was noted in the reaction with $\mathrm{O}\left({ }^{1} D\right)$, that the lifetime of the collision complex is shorter than for the reactions of $\mathrm{S}\left({ }^{1} D\right)$ and $\mathrm{C}\left({ }^{1} D\right)$ with $\mathrm{H}_{2}$, as it has been shown in the classical calculations of collision times and is also reflected in the much smoother resonance structure of the EQM reaction probabilities.

As for the results when initial $j=1$ (bottom panel of Fig.
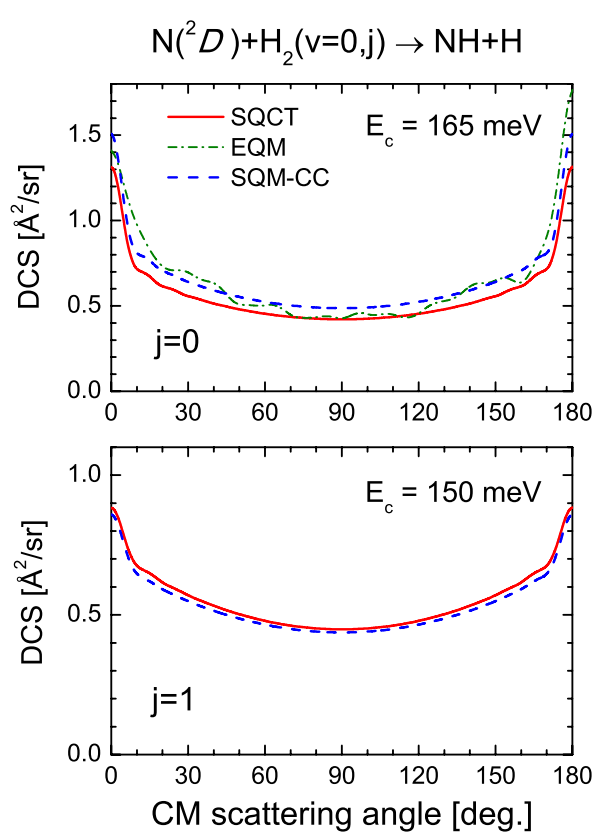

FIG. 11. (Color online) Total (summed over final states) DCS for the $\mathrm{N}\left({ }^{2} D\right)+\mathrm{H}_{2}(v=0, j) \rightarrow \mathrm{NH}+\mathrm{H}$ reaction at $E_{c}=165 \mathrm{meV}$ and $j=0$ (top) and $E_{c}=150 \mathrm{meV}$ and $j=1$ (bottom). Solid line: SQCT; Dashed line: SQM-CC. In the top panel the EQM DCS from Ref. 2 calculated on the PES of Ref. 42 is also represented as a dash-dot line. 

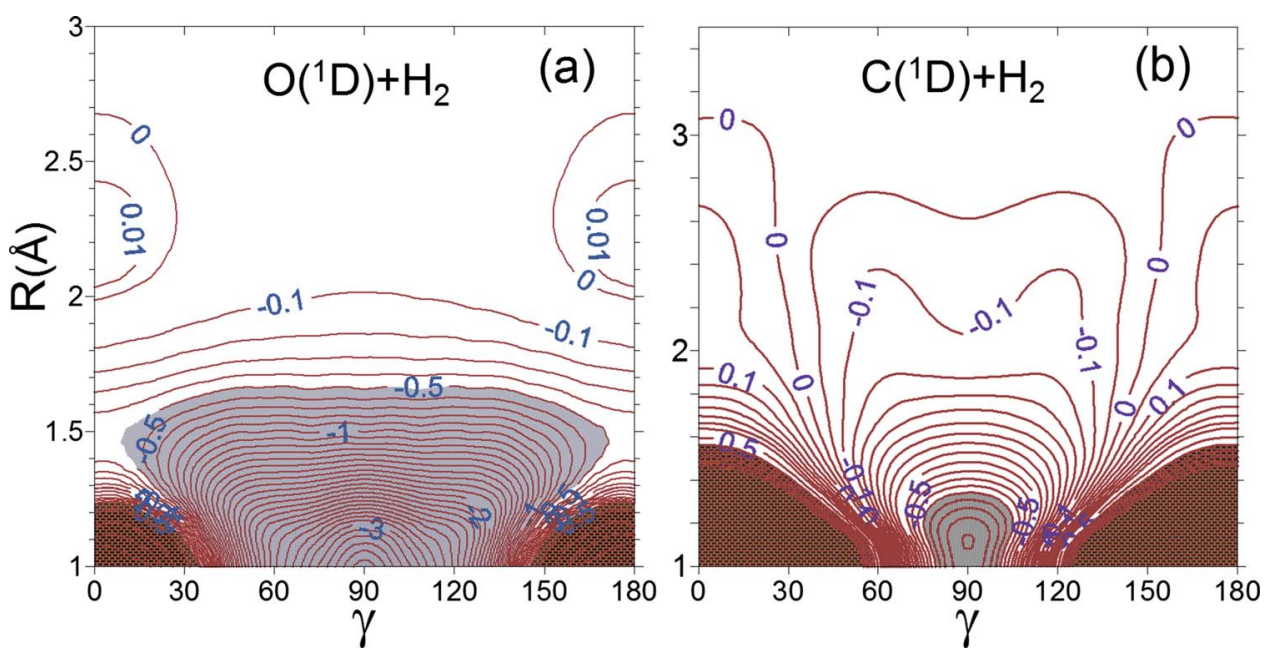

FIG. 12. (Color online) Contour plot (in eV) of the $\mathrm{O}\left({ }^{1} D\right)+\mathrm{H}_{2}$ (a) and $\mathrm{C}\left({ }^{1} D\right)+\mathrm{H}_{2}$ (b) PESs as a function of the $R, \gamma$ Jacobi coordinates of the entrance channel fixing $r$ at the equilibrium distance of the $\mathrm{H}_{2}$ molecule. The dark regions close to $0^{\circ}$ and $180^{\circ}$ are positive potentials above $0.5 \mathrm{eV}$. The gray regions, centered at $90^{\circ}$, represents negative potential values. Trajectories within this region are considered as captured. Contour levels are separated by $0.1 \mathrm{eV}$ in panel (a) (except the $0.01 \mathrm{eV}$ contour), and by $0.05 \mathrm{eV}$ in panel (b).
11), the agreement between the DCS obtained with the SQCT and SQM models is better. Moreover, the effect of the highest $J$ values contributing to the reaction is counterbalanced by larger reactivity in the range $J=12-16$. Consequently, the SQCT DCS, as well as the resulting total cross section, is somewhat larger than that obtained with SQM calculations.

\section{DISCUSSION}

As shown in the previous section, the agreement between the results of the SQCT and SQM model is, in general, remarkably good. For the $\mathrm{C}\left({ }^{1} D\right)+\mathrm{H}_{2}$ and $\mathrm{O}\left({ }^{1} D\right)+\mathrm{H}_{2}$ reactions, as well as for those of $\mathrm{H}^{+}$with $\mathrm{H}_{2}$ and $\mathrm{D}_{2}$ examined in previous works, ${ }^{26,27}$ the results obtained with the two statistical methods are almost indistinguishable even at the highest level of resolution in the product's states. For the reactions of $\mathrm{H}_{2}$ with $\mathrm{S}\left({ }^{1} D\right)$ and $\mathrm{N}\left({ }^{2} D\right)$, although relatively minor, some differences are apparent, especially in the reaction probability. These discrepancies have been shown to be due to the contribution of a relatively narrow range of total angular momenta. It is also clear that the differences in the dynamical observables discussed in the previous section are mostly related to capture probabilities from the initial reagent's states.
Consequently, the origin of these discrepancies and similitudes has to be due to the topology of the respective PES in the entrance channel.

Figures 12 and 13 depict the contour plots of the various PES in Jacobi coordinates, $R$ versus $\gamma$, fixing the $\mathrm{H}_{2}$ internuclear distance $r$ at its equilibrium value. On average, these maps should be a reliable representation of the topology viewed by the reactants approaching the attractive well. In these figures, the capture regions with negative potential values equal of less than the value chosen as capture potential are also marked in light gray color. The strongly repulsive regions are also shown in darker color.

Let us start first with the discussion of the PES for the $\mathrm{O}\left({ }^{1} D\right)+\mathrm{H}_{2}$ system, presented in Fig. 12(a). Except for the small barrier at collinear configurations $(\approx 10 \mathrm{meV})$, the potential is dominated by the attractive well which extends up to $2 \AA$. It is clear that no hindrance exists for the collision energies examined in this work. Even trajectories with attacking angles near collinearity will be captured by the attractive potential in the course of the approach. Hence, the only barrier will be that due to the centrifugal motion which, as in the case of the $\mathrm{H}_{3}^{+}$system, ${ }^{26,27}$ has been shown to be too broad and small enough for the tunneling to play a role. The results obtained by SQCT and SQM-CC should be almost identical as it is indeed the case. The similarities with the
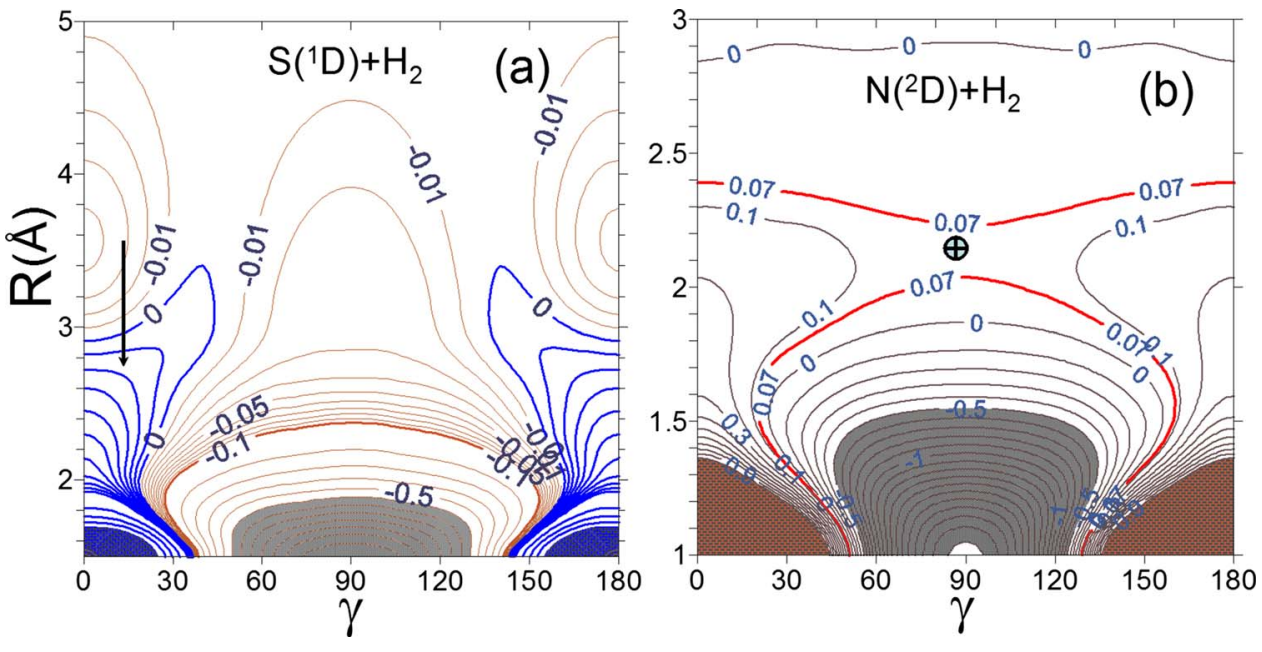

FIG. 13. (Color online) As Fig. 12 but for the $\mathrm{S}\left({ }^{1} D\right)+\mathrm{H}_{2}$ (a) and $\mathrm{N}\left({ }^{2} D\right)+\mathrm{H}_{2}$ (b) reactions. In panel (a) the contours are plotted in steps of $10 \mathrm{meV}$ from -0.1 to $0.1 \mathrm{eV}$, and in steps of $100 \mathrm{meV}$ for smaller and higher values than -0.1 and $0.1 \mathrm{eV}$, respectively. Positive values of the potential are indicated by thicker (blue) lines. Thinner lines (brown) are used for negative contours. The arrow depicts a possible tunneling through the barrier at collinear geometries, which may not be accessible for trajectories with relatively high impact parameters. In panel (b), the thicker (red) line $(0.07 \mathrm{eV})$ is used to show the nearly isotropic barrier. The cross indicates the location of the saddle point for insertion $(0.078 \mathrm{eV})$ at $C_{2 v}$ geometries. 
PES of the $\mathrm{H}_{3}^{+}$system are quite remarkable, as long as it has no practical dynamical barrier. The good agreement between the results from both statistical methods for these reactions is a clear proof that tunneling through a purely centrifugal barrier is not significant.

The $\mathrm{C}\left({ }^{1} D\right)+\mathrm{H}_{2}$ PES is very different, as can be seen in Fig. 12(b), and yet the agreement between QM and QCT statistical calculations is excellent. The region of capture is much more confined than in the case of the $\mathrm{O}\left({ }^{1} D\right)+\mathrm{H}_{2}$ system, and the most remarkable feature is the existence of a collinear barrier which extends up to $R \approx 2.5 \AA$ increasing rapidly with decreasing $R$. However, the shape of this barrier is such that trajectories are likely to be oriented towards the capture region once they hit the repulsive wall. Tunneling is expected to contribute very little to the capture by the potential well. Thus, in spite of the existence of a considerable dynamical collinear barrier and a relatively confined attractive well, the SQCT and SQM-CC results are again practically coincident.

At first glance, apart from the shallow van der Waals wells at collinear configurations, the PES for the $\mathrm{S}\left({ }^{1} D\right)+\mathrm{H}_{2}$, Fig. 13(a), has a topology with mixed resemblances with those discussed for the previous systems. The capture region is rather broad, similar to that of the $\mathrm{OH}_{2}$ system, but in contrast to that system, it is surrounded by a concave barrier relatively confined into the collinear range of approach. A plausible explanation of the observed effect is as follows. Trajectories with a relatively high impact parameter and thus little radial energy but sufficient, however, to overcome a purely centrifugal barrier, can be deflected by this barrier away from the capture region. Even if they were able to cross, they will hit the repulsive part of the potential and they will be rejected back. In the QM case, notwithstanding, tunneling might help to cross through this barrier and reach the attractive well, represented by an arrow in Fig. 13(a). In addition, it is expected that the propagation of the wavepacket would be less sensitive to the specific features of the PES, due to its inherent delocalization. For higher $J$ values (or impact parameters) the centrifugal barrier will decisively limit the possible capture and will diminish the efficiency of tunneling. Under these circumstances, it is expected that the decrease in the classical capture probabilities will take place at high values of $J(J=23-28$, see Fig. 5), but then, for the upper limiting values of $J$, the capture probability will be similar to that of the QM treatment, overall causing a dip in the opacity function. Interestingly, rotational excitation favors the overcoming of the barrier, as it has been shown in various reactive processes. ${ }^{51}$ Seemingly this effect is smaller in the QM calculation and as result of this, the reactivity changes very little in the QM case.

Finally, for the $\mathrm{N}\left({ }^{2} D\right)+\mathrm{H}_{2}$ its PES has a practically isotropic small barrier at a rather long distance $(\approx 2.4 \AA)$. This barrier, however, becomes manifest only at the highest $J$ values $(J=19-22$, see top panel of Fig. 10) where the QM tunneling is apparent. While, the SQCT $P^{J}$ dies off abruptly, there is a tail in the SQM $P^{J}$ that extends up to 3-4 more $J$ quantum numbers. In turn, the dip observed in the $P^{J}$ at smaller values of $J(J=10-17)$ for $j=0$, analogous to that found for the $\mathrm{SH}_{2}$ system, is caused by the concave shape of the collinear barrier partly screening the entrance to the attractive domain of the potential. The $0.1 \mathrm{eV}$ contour extend almost to $60^{\circ}$, preventing the trajectories with small radial energy to be captured. In the QM case, tunneling is effective to cross this barrier, thus increasing the capture probability in this range of impact parameters. When $j$ increases the situation is analogous to the $\mathrm{S}\left({ }^{1} D\right)+\mathrm{H}_{2}$. At the same total energy, the reactivity decreases in both cases, but it does more significantly in the SQM-CC calculations. In the SQCT case, although the maximum $J$ is smaller, the dip at lower $J$ 's disappears and the overall reactivity decreases to a smaller extent. As a result of this, the $j=0$ SQM DCS is larger than the SQCT one over the whole range of scattering angles (see Fig. 11), while for $j=1$, the opposite takes place albeit to a smaller extent. As $j$ increases the differences between SQCT and SQM results become smaller, similarly to what was found in the comparison between EQM and QCT results. ${ }^{25}$

Although the differences between the SQCT and SQM results are very small, thus validating the former method, it has been shown that it is necessary to consider the topology of the PES in some detail. As a general rule, when dynamical barriers exist, tunneling cannot be neglected and this may cause some small but still appreciable differences. The $\mathrm{N}\left({ }^{2} D\right)+\mathrm{H}_{2}$ reaction seems to constitute the process, among the different $\mathrm{A}+\mathrm{H}_{2}$ reactions considered here, for which some more pronounced discrepancies between QCT and QM statistical predictions are found. Although the occurrence of tunneling through the centrifugal barrier was discarded as responsible of the apparent failure of the QCT approach to account for the sharp forward and backward peaks in this kind of insertion reactions, ${ }^{26,30-32}$ the present results suggest that QM effects possibly associated with tunneling through localized dynamical barriers could explain the differences observed in the reaction probabilities at large values of $J$. However, more theoretical work is required to corroborate this explanation. Specifically, incorporation of tunneling effects in QCT calculations may provide a definitive assessment of its role in these reactions.

\section{CONCLUSIONS}

A thorough comparison of the results obtained with the quantum and quasiclassical versions of the statistical model for the prototypical insertion reactions of $\mathrm{C}\left({ }^{1} D\right), \mathrm{O}\left({ }^{1} D\right)$, $\mathrm{S}\left({ }^{1} D\right)$, and $\mathrm{N}\left({ }^{2} D\right)$ excited atoms with $\mathrm{H}_{2}$ is presented in this work. A series of dynamical observables (reaction probabilities, integral, and differential cross sections) with various degrees of resolution in final states have been obtained by both statistical models, and they have been found to be in excellent agreement. The present results for this set of reactions, together with those previously obtained for the $\mathrm{H}^{+}$ $+\mathrm{H}_{2} / \mathrm{D}_{2}$ exchange reactions, clearly proved that SQCT model is very accurate when compared with the corresponding QM version. As shown previously ${ }^{27}$ and corroborated by the present results, the SQCT is sensitive enough to show the relatively minor inaccuracies in the SQM-CS resulting from the decoupling of the projections of the total angular momentum inherent to the CS approximation.

Specifically, for the $\mathrm{C}\left({ }^{1} D\right)+\mathrm{H}_{2}$ and $\mathrm{O}\left({ }^{1} D\right)+\mathrm{H}_{2}$ reactions 
the various results are practically indistinguishable irrespective of their resolution into product's final states. Some differences are, however, observed in the case of the $S\left({ }^{1} D\right)$ $+\mathrm{H}_{2}$ and $\mathrm{N}\left({ }^{2} D\right)+\mathrm{H}_{2}$ systems. Such discrepancies can be explained in terms of the different topologies of their respective PESs. When there are not significant dynamical barriers or those barriers are such that the tunneling is expected to be negligible, the accordance in the results from both methods is nearly perfect. However, when the PES presents some characteristic features, associated with small barriers covering some range of attacking angles susceptible of being overcome by tunneling, some discrepancies are found, usually due to the contribution of narrow ranges of total angular momentum, where the centrifugal energy starts becoming important. Interestingly, the rotational excitation seems to be more effective to promote the capture probabilities, and thus the overall reactivity, in the classical calculations than in the quantum mechanical treatment, something that has been already observed in the comparison of EQM and QCT results for these and other series of reactions.

In some few exemplifying cases, calculations have been carried out disregarding the parity, and the results, entirely analogous to those found for the $\mathrm{H}^{+} \mathrm{H}_{2}$ and $\mathrm{H}^{+} \mathrm{D}_{2}$ systems, confirm that the conservation of parity has to be taken into account in order to obtain sharp forward and backward peaks in the DCS.

The almost perfect agreement between SQCT and accurate SQM-CC (except, perhaps, for the $\mathrm{N}\left({ }^{2} D\right)+\mathrm{H}_{2}$ reaction) does not preclude deviations from the statistical description of these excited atom-hydrogen molecule reactions when compared with experimental data or exact QM results.

\section{ACKNOWLEDGMENTS}

The authors acknowledge funding by the Spanish Ministry of Education and Science (Grant Nos. CTQ200508493-C01-01 and FIS2007-62006).

${ }^{1}$ F. J. Aoiz, L. Bañares, and V. J. Herrero, J. Phys. Chem. A 110, 12546 (2006) and references therein.

${ }^{2}$ P. Honvault and J.-M. Launay, J. Chem. Phys. 111, 6665 (1999).

${ }^{3}$ P. Honvault and J.-M. Launay, J. Chem. Phys. 114, 1057 (2001).

${ }^{4}$ N. Balucani, L. Cartechini, G. Capozza, E. Segoloni, P. Casavecchia, G. G. Volpi, F. J. Aoiz, L. Bañares, P. Honvault, and J.-M. Launay, Phys. Rev. Lett. 89, 013201 (2002).

${ }^{5}$ B. Bussery-Honvault, P. Honvault, and J.-M. Launay, J. Chem. Phys. 115, 10701 (2001).

${ }^{6}$ L. Bañares, F. J. Aoiz, P. Honvault, B. Bussery-Honvault, and J.-M. Launay, J. Chem. Phys. 118, 565 (2003)

${ }^{7}$ P. Honvault and J.-M. Launay, Chem. Phys. Lett. 370, 371 (2003).

${ }^{8}$ L. Bañares, F. J. Aoiz, P. Honvault, and J.-M. Launay, J. Phys. Chem. A 108, 1616 (2004).

${ }^{9}$ K. Yuan, Y. Cheng, X. Liu, S. A. Harich, X. Yang, and D.-H. Zhang, Phys. Rev. Lett. 96, 1032002 (2006)

${ }^{10}$ S. Y. Lin, L. Bañares, and H. Guo, J. Phys. Chem. A 111, 2376 (2007).

${ }^{11}$ Y.-T. Hsu, K. Liu, L. A. Pederson, and G. C. Schatz, J. Chem. Phys. 111, 7921 (1999)
${ }^{12}$ F. J. Aoiz, L. Bañares, J. F. Castillo et al., J. Chem. Phys. 116, 10692 (2002).

${ }^{13}$ N. Balucani, G. Capozza, L. Cartechini et al., Phys. Chem. Chem. Phys. 6, 4967 (2004).

${ }^{14}$ N. Balucani, G. Capozza, E. Segoloni, A. Russo, R. Bobbenkamp, P. Casavecchia, T. González-Lezana, E. J. Rackham, L. Bañares, and F. J. Aoiz, J. Chem. Phys. 122, 234309 (2005).

${ }^{15}$ S.-H. Lee and K. Liu, Appl. Phys. B: Lasers Opt. 71, 627 (2000).

${ }^{16}$ For a recent review see T. González-Lezana, Int. Rev. Phys. Chem. 26, 29 (2007).

${ }^{17}$ P. Pechukas and J. C. Light, J. Chem. Phys. 42, 3281 (1964).

${ }^{18}$ J. C. Light and J. Lin, J. Chem. Phys. 43, 3209 (1965).

${ }^{19}$ J. Lin and J. C. Light, J. Chem. Phys. 45, 2545 (1966).

${ }^{20}$ P. Pechukas, J. C. Light, and C. Rankin, J. Chem. Phys. 44, 794 (1966).

${ }^{21}$ W. H. Miller, J. Chem. Phys. 52, 543 (1970).

${ }^{22}$ E. J. Rackham, F. Huarte-Larrañaga, and D. E. Manolopoulos, Chem. Phys. Lett. 343, 356 (2001).

${ }^{23}$ E. J. Rackham, T. González-Lezana, and D. E. Manolopoulos, J. Chem. Phys. 119, 12895 (2003)

${ }^{24}$ S. Y. Lin and H. Guo, J. Chem. Phys. 120, 9907 (2004).

${ }^{25}$ N. Balucani, P. Casavecchia, L. Bañares, F. J. Aoiz, T. González-Lezana, P. Honvault, and J.-M. Launay, J. Phys. Chem. A 110, 817 (2006).

${ }^{26}$ F. J. Aoiz, V. Sáez-Rábanos, T. González-Lezana, and D. E. Manolopoulos, J. Chem. Phys. 126, 161101 (2007).

${ }^{27}$ F. J. Aoiz, T. González-Lezana, and V. Sáez-Rábanos, J. Chem. Phys. 127, 174109 (2007).

${ }^{28}$ T. González-Lezana, O. Roncero, P. Honvault, J.-M. Launay, N. Bulut, F. J. Aoiz, and L. Bañares, J. Chem. Phys. 125, 094314 (2006).

${ }^{29}$ E. Carmona-Novillo, T. Gonzále-Lezana, O. Roncero, P. Honvault, J.-M. Launay, N. Bulut, F. J. Aoiz, L. Bañares, A. Trottier, and E. Wrede, J. Chem. Phys. 128, 014304 (2008).

${ }^{30}$ P. Larrégaray, L. Bonnet, and J.-C. Rayez, J. Phys. Chem. A 110, 1552 (2006).

${ }^{31}$ L. Bonnet, P. Larrégaray, J.-C. Rayez, and T. González-Lezana, Phys. Chem. Chem. Phys. 8, 3951 (2006).

${ }^{32}$ L. Bonnet, P. Larrégaray, and J.-C. Rayez, Phys. Chem. Chem. Phys. 9, 3228 (2007).

${ }^{33}$ F. J. Aoiz, V. Sáez-Rábanos, B. Martínez-Haya, and T. González-Lezana, J. Chem. Phys. 123, 094101 (2005).

${ }^{34}$ F. J. Aoiz, M. Brouard, C. Eyles, J. F. Castillo, and V. Sáez-Rábanos, J. Chem. Phys. 125, 144105 (2006).

${ }^{35}$ F. J. Aoiz, V. J. Herrero, M. P. de Miranda, and V. Sáez-Rábanos, Phys. Chem. Chem. Phys. 9, 5367 (2007).

${ }^{36}$ L. Bonnet, J. Chem. Phys. 128, 044109 (2008).

${ }^{37}$ L. Bañares, F. J. Aoiz, S. Vázquez, T.-S. Ho, and H. Rabitz, Chem. Phys. Lett. 374, 243 (2003).

${ }^{38}$ T.-S. Ho, T. Hollebeek, H. Rabitz, S. C. Chao, R. T. Skodje, A. S. Zyubin, and A. M. Mebel, J. Chem. Phys. 116, 4124 (2002).

${ }^{39}$ A. J. Dobbyn and P. J. Knowles, Mol. Phys. 91, 1107 (1997).

${ }^{40}$ A. J. Dobbyn and P. J. Knowles, Faraday Discuss. 110, 247 (1998).

${ }^{41}$ T.-S. Ho, H. Rabitz, F. J. Aoiz, L. Bañares, S. Vázquez, and L. B. Harding, J. Chem. Phys. 119, 3063 (2003).

${ }^{42}$ L. A. Pederson, G. C. Schatz, T.-S. Ho, T. Hollebeek, H. Rabitz, L. B. Harding, and G. Lendvay, J. Chem. Phys. 110, 9091 (1999).

${ }^{43}$ S. Y. Lin and H. Guo, J. Phys. Chem. A 108, 10066 (2004).

${ }^{44}$ P. McGuire and D. J. Kouri, J. Chem. Phys. 60, 2488 (1974).

${ }^{45}$ R. T. Pack, J. Chem. Phys. 60, 633 (1974).

${ }^{46}$ J.-M. Launay, J. Phys. B 9, 1823 (1976).

${ }^{47}$ K. McLenithan and D. Secrest, J. Chem. Phys. 80, 2480 (1984).

${ }^{48}$ S. Lin and H. Guo, J. Chem. Phys. 122, 074304 (2005).

${ }^{49}$ A. J. Alexander, F. J. Aoiz, M. Brouard, I. Burak, Y. Fujimura, J. Short, and J. P. Simons, Chem. Phys. Lett. 262, 589 (1996).

${ }^{50}$ S. Lin and H. Guo, J. Chem. Phys. 124, 031101 (2006).

${ }^{51}$ L. Bañares, F. J. Aoiz, T. Gónzalez-Lezana, V. J. Herrero, and I. Tanarro, J. Chem. Phys. 123, 224301 (2005). 